\title{
Impact of intuitive theories on feature recruitment throughout the continuum of expertise
}

\author{
KATHY E. JOHNSON \\ Indiana University-Purdue University, Indianapolis, Indiana \\ and \\ CAROLYN B. MERVIS \\ Emory University, Atlanta, Georgia
}

\begin{abstract}
Expertise in object domains involves both the perceptual learning of the differentiating and higher order features that are indicative of concepts and the elaboration of intuitive theories. Triad-similarity judgments, feature-salience ratings, and verbal protocols were used to investigate the effects of theories on the recruitment of features across different categorization contexts, as well as the degree to which expert categorization skills transferred to less familiar domains. Whereas novices considered features that indicated overall similarity to be more perceptually salient than were modified parts that indicated taxonomic relations, experts found them equally salient. Experts' theories were instrumental in directing feature recruitment in contexts involving identification, image generation, and similarity decisions. Experts' theories also supported the transfer of categorization skills to related, less familiar domains. The relation of mutual dependence between perceptual learning and theory development throughout the continuum of expertise is considered.
\end{abstract}

One of the most striking aspects of expertise in categorical domains is the expert's ability to detect features of which novices are unaware. These features may serve either to differentiate dmong coordinate subordinate-level categories or to indicate higher order categories. The discovery of differentiating features generally occurs in the course of perceptual learning as one is exposed to increasingly precise names for things within the domain (E. J. Gibson, 1969). Although most novices would probably judge unnamed walleyes and saugers to be the same kind of fish, proficient anglers are able to quickly differentiate them on the basis of the degree of speckling present on the dorsal and caudal fins. Features that are indicative of higher order categories generally are learned through the recognition of invariants that are shared by intermediate-level categories within the domain, as when birders come to notice that all songbirds possess a distinctive "three-plus-one" toe pattern. Experts' increased sensitivity to both differentiating

This research was supported by a grant from the National Institute of Child Health and Human Development (HD27042) to the second author and by generous support from the School of Science at IUPUI. We thank Dick Neisser, Howard Rollins, Mike Tomasello, Eugene Winograd, Colleen Seifert, and two anonymous reviewers for their helpful comments on this work. Sharon Hutchins, Amy Eilers, Kelly Switzer, Jennifer Staley, and Joann Helferich assisted with data collection, transcription, and coding. We are especially grateful to the members of the Atlanta and Indianapolis Audobon Societies for their participation. Correspondence and requests for reprints should be addressed to $\mathrm{K}$. Johnson, Psychology Department, IUPUI, LD 124, 402 N. Blackford St., Indianapolis, IN 46202-3275 (e-mail: kjohnso@iupui.edu).

—Accepted by previous editor, Geoffrey R. Loftus and higher order features has implications for many aspects of cognition that depend on conceptual knowledge. For example, recognition of higher order features provides bases for similarity decisions in situations that involve conceptual grouping. Awareness of differentiating features may promote more efficient identification routines and richer mental images involving domain exemplars. It is likely that knowledge of which kinds of features tend to vary among subordinate categories has implications for the induction of novel properties learned in reference to specific domain exemplars.

Although most models of categorization have been based largely on data from tasks involving carefully selected artificial stimuli, the theory-based approach to concepts has focused researchers' attention on complex, realworld categories (Medin, 1989; Murphy \& Medin, 1985). A natural consequence of this approach is an interest in the impact that expertise has on various aspects of conceptual structure and categorization, given that experts presumably have developed maximally elaborated theories. The purpose of the present research was to consider the effects of intermediate and advanced experts' theories on the recruitment of both differentiating and higher order features acquired through perceptual learning. Triad-similarity judgments, ratings of feature salience, and verbal protocols were collected in order to examine differences throughout the continuum of expertise in the features used to relate, identify, and form images of songbird exemplars. A second goal of this research was to consider evidence for the transfer of expert skill in feature detection to related but less familiar domains. We first consider how perceptual learning and domain-specific theories might interact 
to determine experts' use of features across different categorization contexts. We then consider the literature pertaining to the domain specificity of expertise and speculate about how experts' theories might promote the transfer of skill in categorization to related but less familiar domains.

\section{Interaction of Perceptual Learning and Intuitive Theories at Varying Levels of Knowledge}

The perceptual-learning literature is replete with examples of how experience within a domain gives rise to increased discriminatory capabilities (E. J. Gibson, 1969; J. J. Gibson \& E. J. Gibson, 1955; Proctor \& Dutta, 1995). Upon first exposure, perceptually complex sights, smells, tastes, and sounds generally are perceived holistically. Repeated experiences with sensory information promotes the detection of invariants associated with increasingly subtle differentiating features through selective attention. Discrimination may be further enhanced by the presence of linguistic information concerning names and/or differentiating features. Thus, chick sexers become increasingly proficient at differentiating chicks on the basis of cloacal features that generally are not detected by novices (Biederman \& Shiffrar, 1987), and wine tasters learn the features that distinguish clarets from burgundies. Although the acquisition of hierarchically related object categories typically has not been considered from the perspective of perceptual learning, there are obvious implications regarding the development of expertise. To a novice, the basic level of categorization is most salient, simply because objects are readily processed holistically at the basic level-that is, on the basis of overall shape (Neisser, 1987; Rosch, Mervis, Gray, Johnson, \& Boyes-Braem, 1976). The detection of increasingly subtle differentiating features promotes the acquisition of categories at subordinate and, sometimes, even at sub-subordinate levels (Johnson \& Mervis, 1997). Within domains that are integral to one's culture, all individuals may acquire rich domains of subbasic categories, beginning to do so even during the preschool years (Berlin, 1992; Stross, 1973). For less culturally relevant domains, extensive subordinate-category learning typically is relegated to subgroups of experts. Heightened interest is likely to promote discrimination among kinds throughout the continuum of expertise, from preschool dinosaur experts to advanced collectors of rocks and minerals.

We argue that perceptual learning is necessary for the acquisition of expertise in object domains, but it is not sufficient. Also necessary are experts' understanding of why features indicative of particular subordinate categories tend to co-occur and their conceptual understanding of the bases for more abstract categories that share invariant features. These aspects of conceptual knowledge are the hallmarks of the theory-based approach to concepts (Medin, 1989). Perceptual learning provides the data on which experts' theories can operate, but it is their domain-relevant theories that drive the understanding of which kinds of features tend to be shared among domain-specific concepts (Murphy \& Wright, 1984) and which tend to be more distinc- tive. Theories also may promote experts' understanding that it may be more important to attend to certain features rather than to others in particular categorization contexts.

Theories are particularly useful in helping to explain the qualitative reorganization of relations among domainspecific concepts as being a function of knowledge (Carey, 1985; Keil, 1989). Similarity relations that initially were based on relatively superficial features are replaced, with increasing levels of knowledge, by those that are based on more abstract dimensions. For example, Chi, Feltovich, and Glaser (1981) found that, whereas novices in physics sorted physics problems on the basis of keywords or objects presented in the problem statements (e.g., balance beams, inclined planes), experts sorted the same problems on the basis of abstract physical principles. Medin, Lynch, Coley, and Atran (1997) found that groups of experts on trees tended to sort trees into piles that corresponded to goals or ideals that accorded with their particular professions. For example, professional landscapers sorted trees into groups on the basis of utilitarian dimensions (e.g., trees for planting on city streets, flowering ornamental trees, "weed" trees), whereas taxonomists (individuals engaged in teaching about trees and in research pertaining to trees) invariably used taxonomy as the basis for sorting. In a mini-longitudinal study of knowledge acquisition on shorebirds, Johnson and Mervis (1994) found that triadsimilarity judgments based on overall morphological similarity tended to be replaced over time by judgments based on more abstract dimensions, such as diet or mode of locomotion. Thus, weights associated with dimensional bases for similarity relations appear to shift over time as a function of theory development.

Categorization processes are dynamic in that different categorization tasks recruit different kinds of categorical information (Gelman \& Medin, 1993). In tasks involving the "identification function" of categories, overall shape is emphasized (Johnson \& Mervis, 1997; Jones \& Smith, 1993; Smith \& Kemler-Nelson, 1984). In contrast, tasks involving the induction of novel properties have been demonstrated to depend on deep or "core" features that predict category membership, even when membership in a common category is pitted against perceptual similarity (Gelman \& Markman, 1986, 1987). To complicate matters further, individuals' levels of domain-specific knowledge have been found to influence features used in both relating (Chi, Hutchinson, \& Robin, 1989; Johnson \& Mervis, 1994) and identifying (Johnson \& Mervis, 1997; Tanaka \& Taylor, 1991) category exemplars. One goal of the present research was to examine the impact of intermediate and advanced experts' theories on feature recruitment in categorization contexts involving similarity judgments, identification, and the generation of visual images. Data were gathered through both a triad task (Study 1) and through verbal protocols (Study 3 ). To evaluate the extent to which feature recruitment during the triad task was driven by the perceptual salience of features (i.e., through the unmitigated effects of perceptual learning), ratings of feature salience were collected in Study 2. In particular, we 
wished to determine whether the features that were selected as the bases for triad solutions by experts and novices were perceived by experts as equally obvious, or whether experts perceived features that were indicative of their preferred bases for solutions as significantly more obvious. If the features that are indicative of alternative bases for solutions are considered to be equally salient by experts, this would amplify the importance of experts' theories in driving similarity decisions.

\section{Domain Specificity of Expertise}

Controversy exists over two issues that are related to investigations of the domain-specific versus domain-general effects of expertise. First, there is a great deal of variation among researchers in the use of the term domain (Hirschfeld \& Gelman, 1994). Some researchers use this term to refer to extremely broad areas of cognitive competencies, such as the representation of number or causal relations. Others use the term domain to denote theories or coherent clusters of beliefs pertaining to areas such as biology, physics, or music (Carey, 1985; Karmiloff-Smith, 1992). We have adopted the approach frequently seen in the expertise literature, where domain tends to have a considerably narrower focus, typically referring to particular areas of specialization, such as chess, numerical digit span, or dinosaurs (Ericsson \& Smith, 1991).

The second issue pertains to what constitutes evidence for transfer. Within the expertise literature, transfer typically is investigated by trying to determine whether experts are at any advantage when confronted with similar problems drawn from unfamiliar domains. Thus, an individual who has developed an extremely large digit span might be asked to retain comparably long strings of letters (Chase \& Ericsson, 1981), or an expert on chemistry might be presented with problems pertaining to political science (Voss $\&$ Post, 1988). On the other hand, perceptual-learning theorists generally adopt much less stringent criteria for evidence of transfer. In a discrimination-learning task in which the subjects learn by discovering differences among members of a particular set, evidence for the transfer of learning is obtained through subjects' ability to differentiate among previously unseen set members (Pick, 1965). We adopt a definition of transfer that falls somewhere between these two positions. In Studies 1 and 2, experts were given tasks involving exemplars from both the familiar (expert) domain and a domain for which they reported very low levels of knowledge. However, this unfamiliar domain was related to the expert domain in that both were subsumed by the more global domain of biological kinds. Thus, the taxonomic structures of the two domains were isomorphic, although the specific bases for categorizing exemplars from the unfamiliar domain were unknown.

Contemporary views of expertise adopt the stance that expert proficiency is largely restricted to the domain in which knowledge has been acquired (Chi, Glaser, \& Farr, 1988; Glaser, 1987). This is not surprising, given that research on expertise has been devoted predominantly to skill-based domains that are dependent on procedural knowledge. In domains such as these, the strategies that are acquired to increase proficiency are inextricably linked to conceptual knowledge pertaining to the domain. It is unlikely that the strategies mastered by experts on chess could have relevance for the strategies necessary for excellence at backgammon or bridge. Evidence for the transfer of expertise has not yet been sought within domains that are characterized by the knowledge of object categories.

We speculate that experts' theories may well afford some advantage for categorizing objects, even those belonging to unfamiliar domains. Wisniewski and Medin (1994) have demonstrated that individuals' theories are tightly coupled with the extraction and interpretation of features during category learning. Intuitive theories guide the search for evidence in the data and enable people to make inferences as to whether specific features support more abstract features. Although Wisniewski and Medin investigated individuals' learning of categories that frequently contained ambiguous features (children's drawings), we think that the basic principles of their argument might be applied to experts' categorization of considerably less ambiguous object categories from unfamiliar domains. Experts possess theories concerning which types of features tend to be associated with preferred bases for similarity. For example, taxonomic relations within biological domains generally are predicted by modified part features associated with the ecological niche indicative of the related categories. When confronted with categories from unfamiliar domains, experts' theories might guide the extraction of features that align with those preferred within the familiar domain. We investigate this issue through the triad task of Study 1 by using a crossover design in which experts on songbirds and experts on tropical fish solve triads in their own domain, as well as those in a less familiar domain. To the extent that experts generate taxonomic solutions in the novice domain more frequently than do individuals who are novices in both domains, we can speculate that experts' skill at categorization is generalizing beyond the highly familiar domain.

\section{Aims of the Present Research}

In sum, relatively little research has been directed at individuals who have varying levels of expertise in domains that are characterized by natural categories of objects. The primary aim of the present studies was to investigate the impact of experts' theories on feature recruitment across categorization contexts. Features used by advanced and intermediate experts in making triad-similarity judgments in Study 1 were compared with those features the same individuals reported to be relevant for identification, imagery, and conceptual groupings in Study 3. A second goal was to investigate the evidence for the transfer of experts' bases for triad-similarity judgments to less familiar domains. In Study 2, different groups of intermediate and advanced experts rated the salience of the features recruited by experts and novices as the bases for similarity in Study 1. These data were used to investigate the influ- 
ence of feature perceptibility both on similarity judgments and on the degree to which the experts' bases for similarity generalized to the less familiar domain.

\section{STUDY 1 \\ Triad Task}

Conceptual groupings by experts generally differ qualitatively from those by novices (see, e.g., Boster \& Johnson, 1989; Chi et al., 1981; Chi et al., 1989; Johnson \& Mervis, 1994). In particular, novices' categories are based on overall similarities among objects, including shape, size, and color (which we will refer to as morphological similarities), whereas experts' categories are based on more abstract criteria, such as behavioral similarity or taxonomic relationship. The present experiment measured the conceptual groupings by experts and novices through the use of a triad task. In half of the triads, taxonomic relatedness was consistent with overall perceptual similarity, and in half they were in conflict because of the presence of a distractor that was perceptually similar but unrelated to one of the two taxonomically related exemplars. When taxonomy and morphology were consistent, experts and nonexperts were expected to make decisions of similarity rapidly and to choose the same exemplars as being most similar. When taxonomy and morphology were in conflict, experts were expected to base decisions of similarity on taxonomic relations more frequently than nonexperts did, although experts' decisions were expected to be made more slowly. Novices were expected to generate solutions equally quickly to triads of both types, because of their reliance on those features that were indicative of morphological similarity. Subjects were asked to solve triads involving exemplars from the domains of songbirds and tropical freshwater fish. Expert subjects were familiar with only one of these domains. Expert performance in the novice domain permitted examination of the evidence for the generalizability of expert bases for similarity relations.

Method
Subjects
The subjects included two groups of experts on songbirds, one
group of experts on tropical freshwater fish, and one group of
novices. All of the experts were paid for their participation. The
songbird expert group included 12 advanced experts and 8 interme-
diate experts, recruited from a group of 44 individuals who led bird-
ing field trips for the Atlanta Audobon Society. Determinations of
the level of expertise were based on peer ratings. Field trip leaders
were mailed questionnaires on which they were asked to anony-
mously rate each of their peers' knowledge of songbirds on a scale
from 1 to 7 , with 1 being "knows very little about songbirds" and 7
being "one of the most knowledgeable people on songbirds that I've
ever encountered." Each leader also was asked to rate his or her own
knowledge on several other domains (one of which was the novice
domain of tropical freshwater fish) along an identical 7 -point scale.
Thirty-two of the 44 questionnaires were returned. The 12 individ-
uals who had the highest mean peer ratings ( $M=6.47)$, and the 8 in-
dividuals who had the lowest mean peer ratings $(M=5.09$ ) were iso-
lated and treated as advanced experts and intermediate experts,
respectively. One individual from each of the two groups rated his or
her knowledge of tropical freshwater fish as above average (i.e., as

greater than 4) and was replaced. All of the individuals who were selected agreed to participate. The mean self-rating for knowledge of tropical freshwater fish was 1.75 for the advanced experts and 1.88 for the intermediate experts.

The 12 experts on tropical freshwater fish were recruited from employees of tropical fish stores and tropical fish wholesale distributors in the Atlanta area and from the Aquarium Society of Georgia. Expertise again was determined through peer ratings along the same 7-point scale $(M=5.96)$. All of the fish experts indicated that they knew very little about birds $(M$ self-rating $=0.67$; the mean fell below the scale because many individuals rated their knowledge of songbirds as 0 ). Because the fish experts generally were rated by fewer of their peers than were the bird experts, and because expertise on tropical fish typically is characterized by depth of knowledge of one part of the domain (e.g., cichlids) rather than by breadth of knowledge across the whole domain, no comparisons were drawn between the level of expertise possessed by the tropical fish experts and the levels of expertise possessed by the advanced and intermediate experts on songbirds.

The 12 novice undergraduates participated in return for course credit. None of the novices had taken a college-level biology course. The novices' mean self-ratings of knowledge were generated using the same scale as that described above for experts. The mean knowledge rating was 2.42 for songbirds and 3.00 for tropical freshwater fish. The novices' level of interest in the two domains also was quite low. Using the same 7-point scale (with interest replacing the word knowledge), mean interest ratings were 1.33 for songbirds and 1.58 for tropical freshwater fish.

\section{Materials}

The materials included 20 displays of three pictures from each of the songbird and tropical freshwater fish domains. In each of the 40 test displays, individual pictures were presented to scale within a $4 \times 6$ in. $(10.16 \times 15.24 \mathrm{~cm})$ area. Realistic color drawings of birds and color photographs of fish were scanned through a La Cie Silverscanner, edited through Adobe Photoshop, and then presented on a Macintosh IIfx computer with a 13-in. Apple RGB color highresolution monitor. All animals were shown facing to the right, and only male exemplars were included. All bird exemplars resided for at least part of the year in northern Georgia. Fish were photographed in water and presented against a neutral rectangular-shaped background from within the aquarium; birds were presented against a white background. The positioning of the three pictures within each display was randomized. Triads were presented through specially created Authorware programs.

Within each domain, two types of triads were included: (1) triads in which taxonomic relations conflicted with morphological similarity (conflict triads) and (2) triads in which taxonomic relations were consistent with morphological similarity (consistent triads). Conflict triads included two exemplars from the same genus or family that were less morphologically similar to each other than one of the exemplars was to the distractor. Consistent triads included two exemplars from the same genus that were more morphologically similar to each other than either one of the exemplars was to the distractor. Examples of these triads from the domain of songbirds are presented in Figure 1. In both triads, Bird 1 is the distractor and Birds 2 and 3 are taxonomically related. In the conflict triad, Bird I is highly perceptually similar to Bird 2 (they both are yellow and have similarly barred wings), whereas in the consistent triad, Bird 1 is dissimilar to Birds 2 and 3. Degree of similarity was assessed through previously obtained ratings that were collected from adults who were unfamiliar with both birds and fish. Various modifications of the exemplars included in the triads were made until the final set of 40 triads was developed. The criterion for inclusion of a triad in the final set was that the two exemplars that were predicted to be most perceptually similar had to be chosen by a minimum of 9 out of 10 adult raters. 

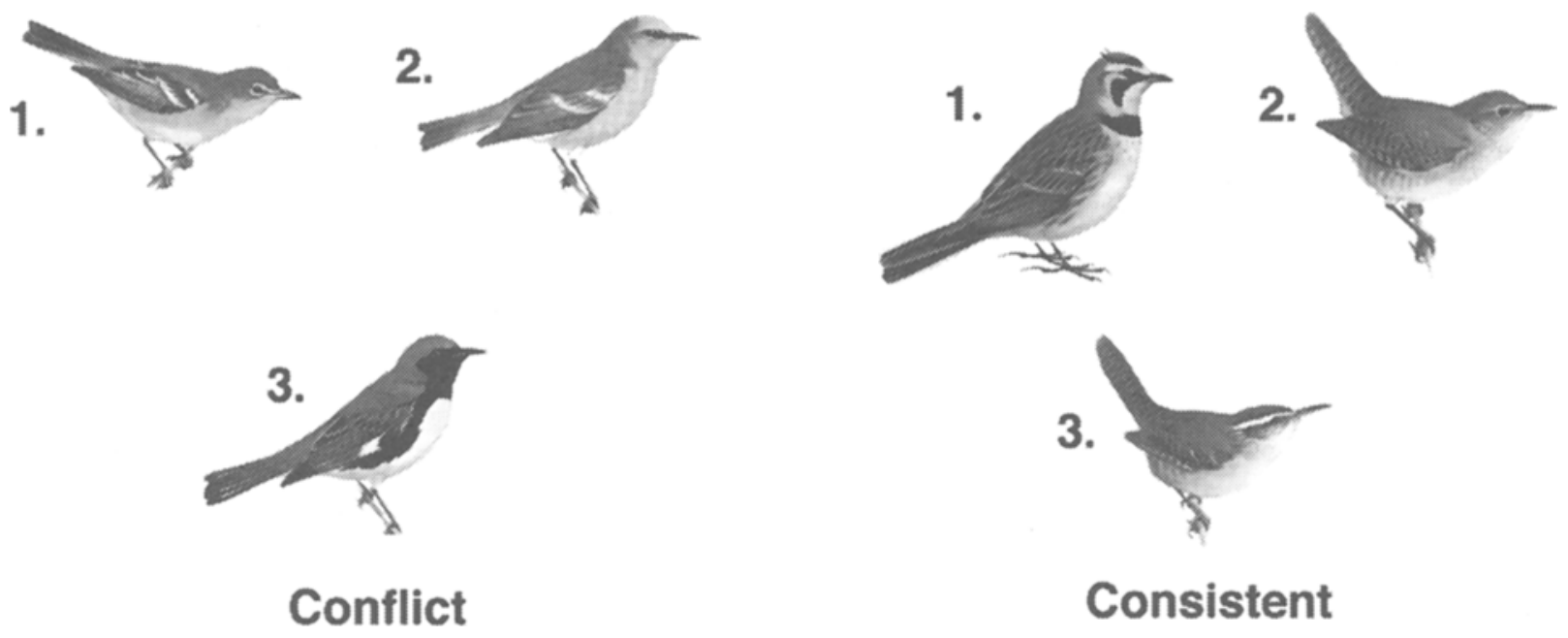

Figure 1. Examples of conflict and consistent songbird triads. Conflict triad: 1, yellow-throated vireo; 2, blue-winged warbler; 3 , black-throated blue warbler. Consistent triad: 1 , horned lark; 2, house wren; 3, Carolina wren. Taxonomic solutions involved selecting exemplars 2 and 3.

Four displays of three color pictures of objects (none of which were birds or fish) also were created for use as practice triads. Each picture was presented in a manner identical to that described above for the test triads. Within each display of three pictures, two of the pictures were obviously more taxonomically related than was a third. In two of these fous triads, taxonomic relationship was consistent with overall morphological similarity (e.g., tree-flower-butterfly), and in two triads taxonomy conflicted with overall morphology (e.g., doughnut-tire-cookie).

\section{Procedure}

The subjects were first administered the four practice triads. In reference to each triad, subjects were asked to "touch the two that are most like the same kind of thing." A Microtouch screen fitted to the front of the computer monitor recorded which pictures were touched. No errors were made by the subjects in the three expert groups. Three novices each produced one error in which the doughnut was paired with the tire on the doughnut-tire-cookie triad. When the triad was repeated, all 3 individuals solved it correctly. The subjects then were presented with a series of the 20 triads for each of the two domains. Two random orders of the triads within each domain were created, and half of the subjects within each group received each order. The order in which the triads from each of the two domains was presented to the subjects also was counterbalanced, so that half of the subjects in each group first received triads from the songbird domain, and half first received triads from the tropical fish domain. The instructions given for the practice triads were presented on the monitor for each of the 40 test triads. During the administration of the test triads, a short break occurred between the presentation of the triads from the first domain and the presentation of the triads from the second domain. After completing each triad, the subjects were asked to press $C$ for "continue" if they wanted to move on to the following triad or R for "return" if they wanted to change their solution to the current triad. Response times were measured, through Authorware, from the time that the three pictures were presented until the subject pressed $\mathrm{C}$. The basis for each triad solution was recorded by the Authorware program as each of the triads was solved. Solutions in which the two exemplars from the same genus were chosen were coded as "taxonomic." Solutions involving the selection of one exemplar from the target genus and the morphologically dissimilar distractor were coded as "other." Solutions to conflict triads in which one exemplar from the target genus and the morphologically similar distractor were chosen were coded as "morphological."

\section{Results}

\section{Solution Type}

In conflict triads, three solutions were possible: taxonomic, morphological, and "other." In consistent triads, only taxonomic and "other" solutions could be generated. The mean proportions of times in which each of the types of solutions was made for each set of triads are summarized in Table 1 . Subjects from all four groups nearly always made taxonomic solutions to consistent triads. However, solutions to conflict triads yielded clear differences as a function of level of expertise.

It is possible that experts who were first presented with triads from their domain of expertise would be more likely to solve triads from the novice domain taxonomically than they would be if the triads from the unfamiliar domain were presented first. This is because expert taxonomic solutions, particularly for conflict triads, require that attention be directed to more subtle differentiating features (e.g., the shape of the mouth or beak) that are correlated with taxonomic membership; such a response set might carry over to the triads from the less familiar domain. In order to investigate this possibility, the numbers of taxonomic solutions (out of 10 ) produced by the three groups of experts were analyzed in a $3 \times 2 \times 2 \times 2$ mixed analysis of variance (ANOVA), with knowledge group and order of triad presentation (expert domain presented first vs. second) as between-groups variables and both domain and triad type as within-group variables. The main effect of order of presentation was not significant, nor were any interactions involving order of presentation. Thus, the experts who were presented with the triads from the familiar domain first were not biased by their solution sets 
Table 1

Mean Proportions of Types of Solutions Across Triads

\begin{tabular}{|c|c|c|c|c|}
\hline \multirow[b]{2}{*}{ Solution Type } & \multicolumn{4}{|c|}{ Knowledge Group } \\
\hline & $\begin{array}{c}\text { Advanced } \\
\text { Bird Experts }\end{array}$ & $\begin{array}{l}\text { Intermediate } \\
\text { Bird Experts }\end{array}$ & Fish Experts & Novices \\
\hline \multicolumn{5}{|c|}{ Songbird Triads } \\
\hline \multicolumn{5}{|l|}{ Conflict Triads } \\
\hline Taxonomic & .91 & .74 & .38 & .09 \\
\hline Morphological & .06 & .19 & .58 & .88 \\
\hline Other & .03 & .07 & .04 & .03 \\
\hline \multicolumn{5}{|l|}{ Consistent Triads } \\
\hline Taxonomic & 1.00 & 1.00 & .94 & .98 \\
\hline Other & .00 & .00 & .06 & .02 \\
\hline \multicolumn{5}{|c|}{ Tropical Fish Triads } \\
\hline \multicolumn{5}{|l|}{ Conflict Triads } \\
\hline Taxonomic & .52 & .50 & .88 & .19 \\
\hline Morphological & .46 & .48 & .09 & .74 \\
\hline Other & .02 & .02 & .03 & .07 \\
\hline \multicolumn{5}{|l|}{ Consistent Triads } \\
\hline Taxonomic & .95 & .94 & 1.00 & .96 \\
\hline Other & .05 & .06 & .00 & .04 \\
\hline
\end{tabular}

when presented with the triads from the unfamiliar domain. Consequently, the order of presentation was not considered in subsequent analyses.

The numbers of taxonomic solutions made by the subjects across the 40 triads were analyzed in a $4 \times 2 \times 2$ mixed ANOVA, with knowledge group as the betweengroups factor and both domain and triad type as withingroup factors. The main effects of knowledge group $[F(3,40)=60.760, p<.0001]$ and triad type $[F(1,40)=$ $603.522, p<.0001]$ were significant. The novices made significantly fewer taxonomic solutions than did any of the three expert groups, and the subjects produced significantly more taxonomic solutions for consistent triads than they did for conflict triads. There were significant interactions between knowledge group and domain $[F(3,40)=$ $38.372, p<.0001]$ and between knowledge group and triad type $[F(3,40)=50.755, p<.0001]$. These need to be interpreted in the light of a significant three-way knowledge group $\times$ domain $\times$ triad type interaction $[F(3,40)=$ $21.459, p<.0001]$. Within the expert domain, both the advanced bird experts and the fish experts made comparable numbers of taxonomic solutions across conflict and consistent triads. However, Tukey (HSD) $t$ tests indicated that the intermediate bird experts made significantly more taxonomic solutions to consistent triads than to conflict triads $(p=.05)$. Furthermore, the intermediate bird experts made significantly fewer taxonomic solutions to conflict triads within the familiar domain than did both the advanced bird experts $(p<.05)$ and the fish experts $(p<.05)$. Individuals who are at more advanced levels of expertise appear more likely to base similarity judgments on features relevant to taxonomic membership than do individuals with less knowledge.

To investigate whether the bases for the experts' triad solutions would generalize to the less familiar domain, separate mixed 4 (knowledge group) $\times 2$ (triad type) ANOVAs were conducted on the taxonomic solutions generated within the bird domain and within the fish domain. Both domains yielded parallel significant interactions between knowledge group and triad type [bird domain: $F(3,40)=$ 44.74, $p<.001$; fish domain: $F(3,40)=32.24, p<.001]$. No differences emerged across groups in terms of responses to consistent triads within either domain. However, responses to conflict triads revealed some evidence that expert solutions did generalize to less familiar categories. While morphological solutions predominated among novices, Tukey (HSD) tests revealed that experts on the contrasting domain generated significantly more taxonomic solutions for the triads involving unfamiliar exemplars than did the novices, who knew little about either domain [bird domain: novices vs. fish experts $(p<.001)$; fish domain: novices vs. advanced bird experts $(p<.001)$; novices vs. intermediate bird experts $(p<.05)] .{ }^{1}$ Several of the experts commented that they knew virtually nothing about the novice domain and were very unsure as to how triads from that domain should be solved. Nevertheless, the experts consistently produced more taxonomic solutions to triads from the unfamiliar domain than did the novices.

\section{Response Time}

We were specifically interested in two issues pertaining to the speed with which triad solutions were generated. First, we wondered whether the individuals' response times would be significantly slowed by the presence of a distractor with high levels of overall similarity to one of the related exemplars. Mean response times were generated for each subject across the 10 triads of each type and compared in paired $t$ tests conducted separately for each knowledge group. Separate $t$ tests were conducted for triads involving birds and for triads involving fish. The group means from these analyses are presented in Figure 2. For the triads involving birds, all expert groups performed significantly faster on the consistent triads than they did on the conflict triads $(p<.05)$. The difference in the novices' 


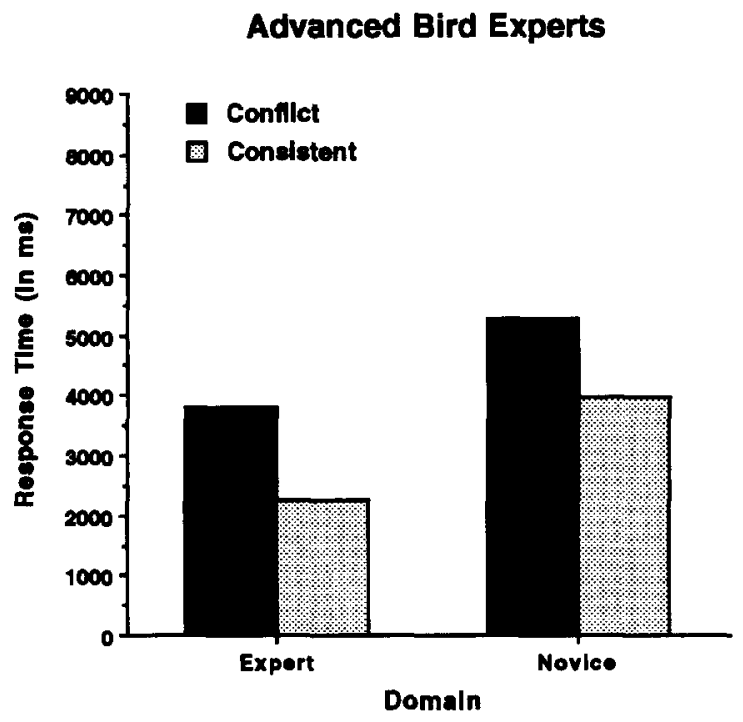

Intermedlate BIrd Experts

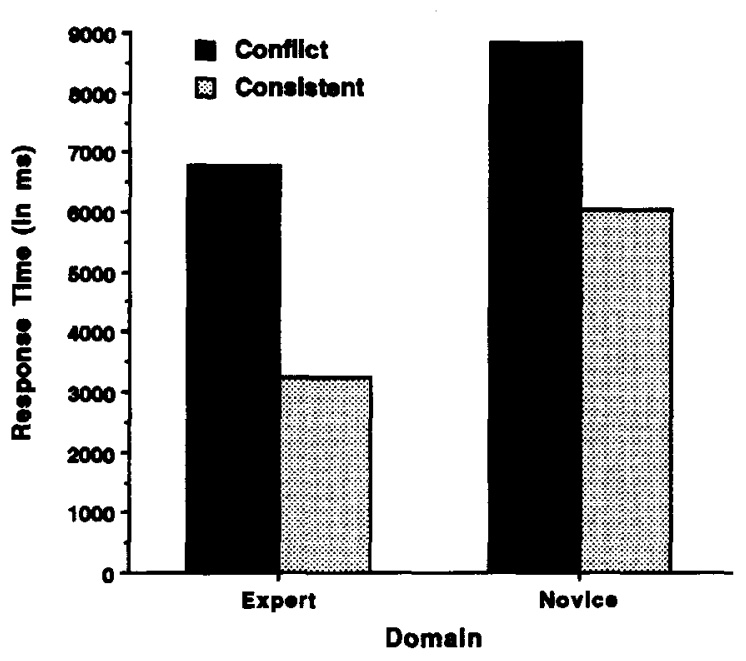

Fish Experts

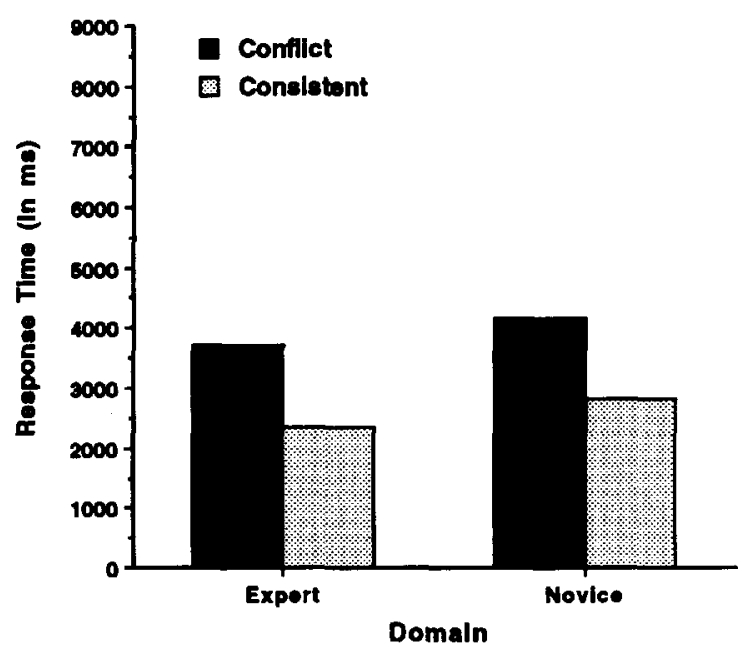

Novices

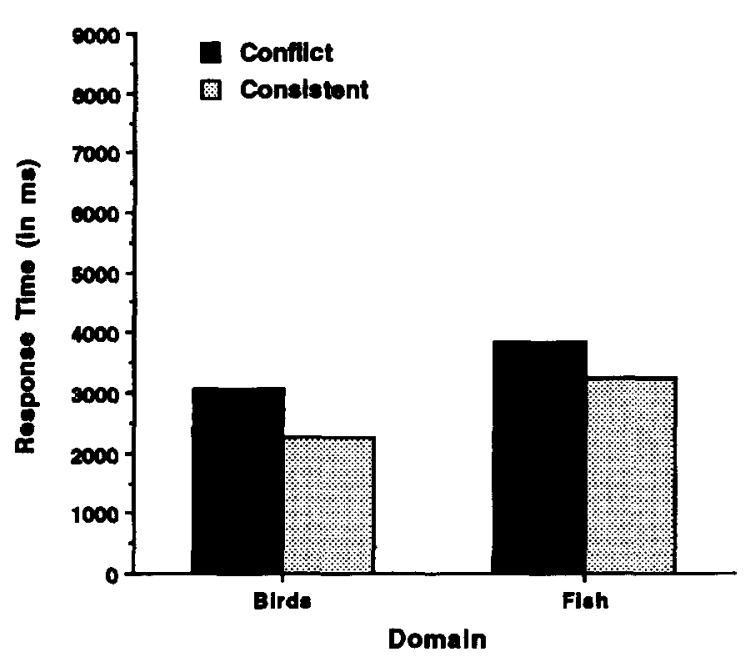

Figure 2. Mean response times across types of triads.

solution times across the two triad types only approached significance $[t(11)=1.99, p=.07]$. For the triads involving fish, only the fish experts $[t(11)=5.73, p<.001]$ and the advanced bird experts $[t(11)=3.42, p<.01]$ generated significantly faster solutions for consistent triads. Differences across triad types were not significant for the intermediate bird experts or the novices. These results suggest that, within the familiar domain, the experts' solutions to the consistent triads were facilitated by the presence of features that were indicative of overall morphological similarity. This pattern generalized to the less familiar domain only for the advanced experts and the fish expertsthat is, the individuals who generated the greatest number of taxonomic solutions for the triads involving unfamiliar stimuli. The novices' response times presumably varied less across the two triad types because they were consistently recruiting the same types of features throughout the task.

Second, we were interested in whether the groups would differ in the degree to which they were affected by the presence of the similar distractor in the conflict triads. In particular, we wondered whether the experts would be less affected by the distractor when they were solving triads within their own domain of expertise than they would be when solving triads involving less familiar exemplars. Separate one-factor ANOVAs were performed on response times to the conflict triads for each of the two domains. Both analyses yielded significant main effects of knowledge group [birds, $F(3,40)=5.54, p<.01$; fish, $F(3,40)=7.73, p<.001]$. Post hoc $t$ tests revealed that, within both domains, the intermediate bird experts gener- 
ated solutions significantly more slowly than did the three other groups. No other differences among groups were significant. ${ }^{2}$ These results suggest that the advanced bird experts and the fish experts generally were not more immune to the effects of the similar distractor within their own domains. They also indicate a quantitative advantage of the advanced bird experts over the intermediate bird experts in terms of the speed with which such decisions are generated. Finally, it is interesting to note that the advanced bird experts and the fish experts were able to generate taxonomic solutions to the triads within the familiar domain just as rapidly as novices generated morphological solutions. The intermediate experts' considerably slower solutions indicate that the advanced experts' speed probably was gained only after a transitional period involving more prolonged weightings of potential candidates for similarity decisions.

\section{Discussion}

Individuals within all three expert groups tended to recruit features indicative of taxonomic relatedness when generating similarity decisions, whereas the novices invariably based their similarity judgments on overall perceptual similarity. Although there were few differences across the four groups in the number of taxonomic solutions made to the consistent triads, clear differences emerged as a function of expertise in the number of taxonomic solutions made to the conflict triads. When presented with triads in which morphological similarity and taxonomic relationship were orthogonal, the advanced experts on birds and the experts on fish were significantly more likely than were the intermediate bird experts, who in turn were significantly more likely than were the novices, to choose taxonomic relations as the most salient basis for their solutions. The intermediate experts were significantly slower than were the other groups in generating solutions to both types of triads, and all experts tended to solve the conflict triads more slowly than they solved the consistent triads. The response-time data suggest that, even though experts may generally recruit modified part features when generating similarity decisions (even on consistent triads), their decisions are at least partially facilitated by the availability of correlated features that are indicative of overall perceptual similarity. In the conflict triads, the experts responded more slowly, presumably because these solutions required the experts to search across multiple bases for alignment when making decisions of similarity (Medin, Goldstone, \& Gentner, 1993).

These results converge with the basic finding in the expertise literature that the conceptual groupings of experts and novices typically differ in terms of the abstractness of the relations considered to be most salient (Chi et al., 1981; Johnson \& Mervis, 1994; Scott \& Mervis, 1993). Taxonomic relations are less "perceptible" than relations based on similarity of overall shape, color, or size. However, taxonomic relations, like most other "abstract" relations within object domains (e.g., diet, defense mechanisms), are correlated with subtle perceptual features that indi- viduals with high levels of knowledge are especially likely to notice. Thus, the advanced and intermediate experts may have been basing their similarity decisions in part on perceptible features that were different from and more subtle than those on which the novices were basing theirs, rather than solely on the more abstract conceptual basis of shared taxonomic category membership. This interpretation is supported by analyses concerning the generalization of expert patterns of similarity judgments to triads from the novice domain.

All three groups of experts produced significantly more taxonomic solutions to the triads from the unfamiliar domain than did the novices, who knew little about either domain. This result suggests that some aspect of the experts' domain-specific theoretical knowledge may have guided the recruitment of modified part features in the less familiar biological domain that aligned with those preferred within the familiar domain. This conclusion is well supported by comments the subjects made when they were debriefed. Many of the experts indicated that, when encountering triads from the novice domain, they focused on relatively subtle features (such as beak or fin shape) and generated their solutions in accord with which exemplars were most similar in terms of these features. When none of these more subtle types of similarities was obvious for a particular triad, the subjects fell back on more global perceptual bases for similarity, such as color, size, and overall shape. This result provides preliminary evidence that expert skill at categorization may transfer beyond the expert domain. It is interesting that differences between the advanced and the intermediate experts did not emerge in the frequency with which taxonomic solutions were generated for fish triads, despite the clear advantage the advanced experts displayed within the familiar domain. Perhaps theories are capable of impacting similarity decisions within less familiar domains even before an extremely high quantity of domain-specific categorical knowledge has accrued. The intermediate bird experts reported that they had been birding, on average, for 15 years. This presumably would have been sufficient time for developing theories concerning linkages between certain types of perceptual features (e.g., mouth shape, head shape) and more abstract behavioral features that are indicative of membership in a particular ecological niche.

The results from the triad task indicated that individuals who possess high levels of domain-specific knowledge tend to recruit subtle perceptual features that are indicative of taxonomic membership as bases for similarity. However, it is still not clear to what extent perceptual learning influenced the experts' solutions to the conflict triads. It is possible that the experts were driven purely by perceptual salience, in that features indicative of modified parts had, as a function of perceptual learning, become more salient than had morphological features. This would relegate theoretical knowledge to a less prominent role in feature recruitment. On the other hand, if the experts considered morphological features to be more salient than taxonomic features (or if both types of features were con- 
sidered to be equally salient), the role of theories in directing similarity decisions would be amplified. In Study 2, feature salience ratings were collected from different groups of bird experts and novices to investigate this issue, as well as to provide an index of the degree to which feature-salience may have contributed to the obtained knowledge-transfer effect. We expected that all groups of subjects would generate comparable salience ratings for morphological features. However, the experts on songbirds were expected to judge modified part features of both birds and fish as more salient than were novices.

\section{STUDY 2 Feature Salience Rating Task}

In the present study, features indicative of morphological and taxonomic solutions to the triads in Study 1 were generated by a group of novice pilot subjects and then were rated by different groups of intermediate and advanced bird experts and novices.

\begin{abstract}
Method
Subjects

The subjects were recruited from the Indianapolis area through methods similar to those used in the previous study. The experts on songbirds were assigned to advanced or intermediate expert groups on the basis of peer ratings of levels of knowledge. Questionnaires were mailed to 31 birders who either led field trips or regularly attended field trips sponsored by the Indianapolis Audobon Society and the Indianapolis Parks Department. The individuals were asked to anonymously rate their peers' level of proficiency at visually identifying songbirds. Ratings were made on the same 7-point scale described for Study 1. Of the 31 questionnaires mailed, 27 were returned. Two additional individuals were recruited once the study began, on the basis of peer referrals. Both of these individuals were assigned ratings by study subjects when they came into the lab. Thirty-one of the 33 individuals who had received peer ratings participated in the study. The 12 individuals who had the highest peer ratings were considered advanced experts $(M=6.57)$, and the remaining 19 individuals were considered intermediate experts $(M=5.31)$. The mean self-ratings of knowledge of tropical freshwater fish were 2.98 for the advanced experts and 2.84 for the intermediate experts. The experts were given gift certificates to local wild bird supply stores in return for their participation. The novice group included 20 undergraduate students enrolled in psychology courses at IUPUI who participated for course credit. All of the novices rated their knowledge of both songbirds $(M=2.07)$ and tropical fish $(M=1.76)$ as low. Eight additional novices participated in the feature-generation phase of the study, as described below.
\end{abstract}

\section{Materials}

Eight groups of three songbirds and 10 groups of three tropical fish were assembled that were isomorphic to the conflict triads in the previous study. Two triads from Study 1 involving the morphologically similar songbirds indigo bunting and blue grosbeak were not included, because we were informed by songbird experts that recent DNA evidence had revealed a close taxonomic relation between the two birds, despite their inclusion in different genera. ${ }^{3}$ In addition, the two conflict triads within each domain that had generated the largest numbers of solutions coded as "other" (in which an exemplar of the target genus and the morphologically dissimilar distractor were selected as most like the same kind of thing) were replaced by triads involving morphologically similar pairs that had previously been used, along with a novel exemplar that was taxonomically re- lated to one of the previously used exemplars. The composition of the resulting 8 songbird triads and 10 tropical fish triads is listed in the first columns of Appendices A and B. The first two exemplars listed for each triad were taxonomically related, and the second and third exemplars listed were similar in terms of overall morphology. Triads were presented through an Authorware program on a 13-in. Apple RGB color high-resolution monitor in the same manner as that described for Study 1.

\section{Procedure}

Feature generation phase. In order to generate sets of features indicative of morphological similarity or taxonomic relations for each triad, eight novice pilot subjects were presented with each of the 18 triads. Although reliance on novice feature generations runs the risk of creating lists of features that are not valid for experts, we were concerned that experts' generation of such a list (particularly for morphological solutions) would be biased by their knowledge of the categories to which the exemplars belonged. Norman, Brooks, Coblentz, and Babcook (1992) have provided evidence within the domain of radiology that experts' feature identifications are partially dependent on category identifications. We were concerned that the availability of category knowledge would influence which features were listed, ultimately leading to the generation of features that were simply unavailable to novices.

The exemplars within each triad were numbered, and the subjects were asked to indicate similarities for the morphologically based and the taxonomically based pairs within each triad (e.g., "How are birds 2 and 3 like the same kind of thing?"). Features that were mentioned by 7 or more novices for each type of pair were used in the featuresalience rating task and are listed in the second and third columns of Appendices A and B. The accuracy of the resulting set of features was verified through published reference materials on songbirds and tropical fish. The accuracy of the features involving birds was independently verified by two experienced birders who did not participate in either Study 1 or Study 2. Booklets were created that contained lists of the features corresponding to each triad. Two different orders of triads were created, and half of the subjects in each group received each order. In addition, half of the subjects in each group were presented with triads from the songbird domain first, and half were presented with triads from the tropical fish domain first. The features corresponding to particular triads were listed in a random order.

Rating phase. The booklets were distributed to the subjects, and the experimenter explained the procedure for making ratings. At the top of each page of the booklet, a 7-point rating scale was presented, where 1 was "very difficult to notice," 4 was "moderately noticeable," and 7 was "very noticeable." The subjects were instructed to rate how obvious or noticeable they perceived each of the specified characteristics to be. The exemplars within each triad were numbered, and the subjects made ratings in response to statements such as "Birds 2 and 3 share the same kind of bill" or "Fish 1 and 2 share the same kind of striped pattern." Features that involved body parts whose names might have been unfamiliar to the novices were accompanied by descriptors in order to ensure that the correct features were rated (e.g., "ventral fin" was indicated as the fin under the fish's body). The subjects proceeded through the lists of features at their own pace, and a short break occurred between the two domains.

\section{Results}

Mean ratings for features that were indicative of overall morphological similarity and for features that were indicative of taxonomic relatedness were calculated for each subject by averaging across ratings that were generated for features of that type within each of the two domains. These mean ratings were then compared across groups in a $3 \times 2 \times 2$ mixed ANOVA with level of knowledge as the 


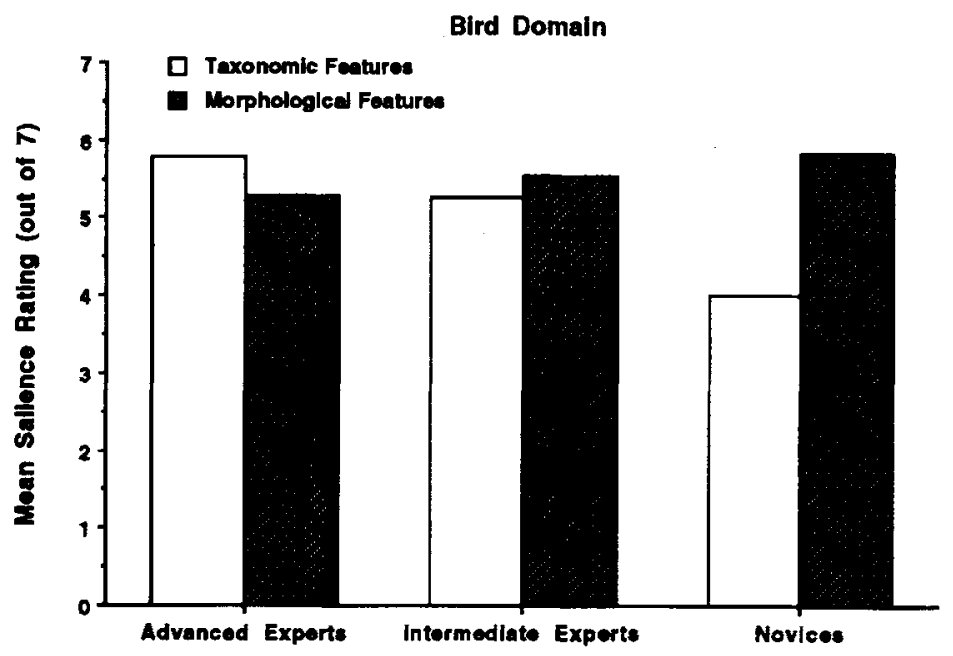

Flah Domaln

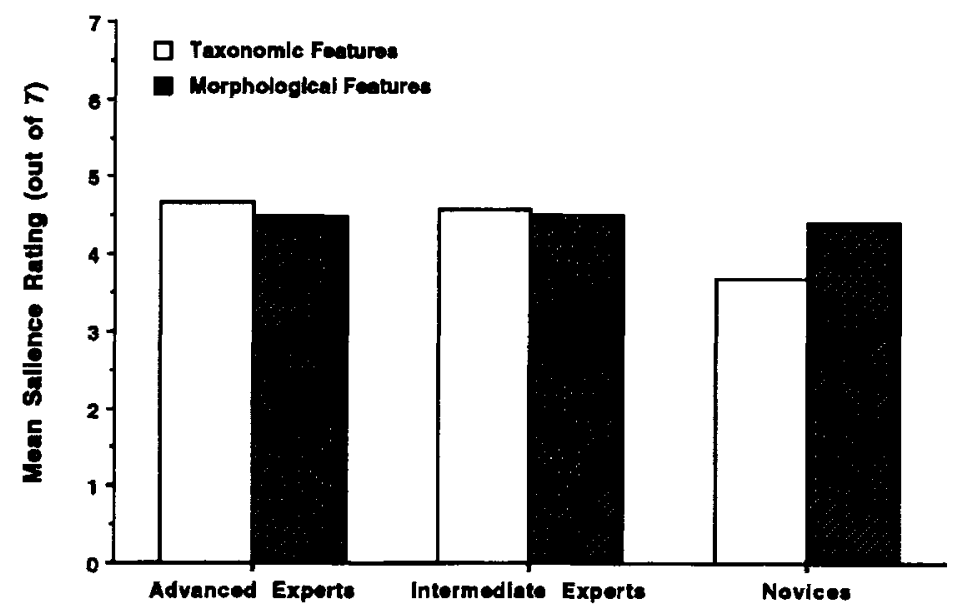

Figure 3. Mean salience ratings generated across the domains of songbirds and tropical fish.

between-groups factor and both domain and feature type as within-group factors. The means for this analysis are depicted in Figure 3 . All main effects were significant: level of knowledge $[F(2,48)=3.60, p<.05]$, domain $[F(1,48)=$ $79.43, p<.001]$, and feature type $[F(1,48)=9.95, p<.01]$. Overall, the novices perceived the features as being less obvious than did the experts, the features of fish were perceived as being less obvious than the features of birds, and the features that were indicative of taxonomic relations were perceived as being less obvious than the features that were related to overall morphological similarity. These effects were tempered by a significant knowledge level $X$ domain $\times$ feature type interaction $[F(2,48)=6.26, p<.01]$, which was explored further through the separate mixed and repeated measures ANOVAs described below.

\section{Ratings of Songbird Features}

Mean ratings of the taxonomic and morphological features pertaining to pairs of songbirds were analyzed in a $3 \times 2$ mixed ANOVA, with level of knowledge as the betweengroups factor and feature type as the within-group factor. The main effects of level of knowledge $[F(2,48)=4.29$, $p<.05]$ and feature type $[F(1,48)=12.05, p<.01]$ were both significant, as well as the level of knowledge $\times$ feature type interaction $[F(2,48)=11.41, p<.001]$. While the three groups did not differ in terms of their salience ratings of morphological features, Tukey (HSD) tests indicated that the advanced and the intermediate experts rated taxonomic features as significantly more obvious than did the novices $(p<.001)$. There were no significant differences between the ratings of the advanced and the intermediate experts. Finally, separate repeated measures ANOVAs conducted for each group revealed that, whereas the novices rated morphological features as significantly more obvious than taxonomic features $[F(1,19)=64.67, p<.0001]$, neither group of experts yielded a significant main effect of feature type - that is, the advanced and intermediate experts on songbirds considered features that were indicative 
Table 2

Interview Questions

\begin{tabular}{ll}
\hline Categorization Context & Format of Question \\
\hline Identification & (1) How do you know an $X$ when you see one? \\
Visual imagery & (2) When you imagine an $X$ what position is it in? \\
Similarity relations & (3) What is it doing? \\
& (4) What $X$ ? Why? Unds are most likely related to $X$ ?
\end{tabular}

Note- " $X$ " referred to each of the 10 category names, yielding a total of 40 responses.

of taxonomic relatedness and features that were indicative of morphological similarity to be equally obvious.

\section{Ratings of Tropical Fish Features}

In order to address the impact of feature salience on the knowledge-transfer effect found in Study 1, parallel analyses were conducted on ratings generated for the domain of tropical fish. Mean ratings for the two types of features were first compared in a $3 \times 2$ mixed ANOVA, with level of knowledge as the between-groups factor and feature type as the within-group factor. There was a significant level of knowledge $\times$ feature type interaction $[F(2,48)=7.02$, $p<.01]$, because of the fact that the novices differentiated between the two types of features in their ratings significantly more than did either group of songbird experts. Indeed, repeated measures ANOVAs conducted separately for each group paralleled the results from the songbird domain: Whereas the novices rated morphological features as significantly more obvious than were taxonomic features $[F(1,19=12.97, p<.01]$, the advanced and intermediate experts rated the two types of features as equally obvious. Separate one-factor ANOVAs conducted for each of the two types of features revealed that, whereas there was no effect of level of knowledge for ratings of morphological features, a main effect of level of knowledge emerged for features indicative of taxonomic relations $[F(2,48)=4.85, p=.01]$. In accordance with the triad task data from Study 1, Tukey (HSD) tests indicated that both the advanced and the intermediate experts on songbirds rated features that were indicative of taxonomic relations among tropical fish as significantly more obvious than did individuals who were novices on both songbirds and tropical fish $(p=.01)$.

\section{Discussion}

The results from the previous two studies converge to suggest that one consequence of expertise is an enhancement of the degree of perceptual salience afforded to relatively subtle features that are correlated with taxonomic relatedness. Whereas novices rate features indicative of overall similarity as more salient than modified part features, experts consider them to be equally salient. ${ }^{4}$ The finding that the intermediate and the advanced bird experts did not differ in their ratings of fish features converges nicely with the lack of a level-of-expertise effect on the evidence for knowledge transfer in Study 1. Again, moderate levels of knowledge are sufficient for theories to impact the perceived salience of features within a less familiar domain.
The results from Study 2 also suggest a mutually dependent relation between enhanced perceptual discrimination and theoretical knowledge throughout the continuum of expertise. Perceptual learning enhances the discriminability of perceptible features that are indicative of object categories to the point where there are a number of equally salient features indicative of sub-basic categories. Thus, theories are essential for driving similarity decisions. At the same time, theories were found to influence the experts' perceptions of salience within the unfamiliar domain. The bird experts presumably possessed poorly elaborated theories specific to the domain of fish. Nevertheless, both intermediate and advanced bird experts rated modified part features of fish as significantly more salient than novices did. This suggests that not only did the experts' theories help to guide their attention to perceptual features in the novice domain that were isomorphic to those linked with more abstract features in the expert domain, but they also helped to amplify the perceptual salience of those features.

Further research is needed to investigate the relative contributions of knowledge and other individual characteristics in predicting degrees of feature perceptibility. Recent research on the categorization performance of child and adult experts has revealed significant relations between expertise and levels of both verbal and nonverbal intelligence (Alexander, Johnson, Lazar, \& Meyer, 1998; Johnson \& Eilers, 1996; Johnson \& Mervis, 1994). If brighter individuals tend to develop intense interests in object domains more frequently than do individuals who are less bright, it is possible that some of the effects of expertise we have discovered are due to the fact that brighter individuals are better at encoding features and detecting invariants than are individuals who are less bright. Such an advantage would greatly facilitate knowledge acquisition throughout the entire continuum of expertise. Generalization of expert skill at feature detection to less familiar domains might be partially afforded through a more analytic style of perception in general, rather than simply through the brute force of domain-related theories. We recently found that subordinate-category names are more readily learned by 3 -year-olds who possess a more reflective cognitive style and who already have acquired large numbers of subordinate category names (Mervis, Johnson, \& Mervis, 1994). Evidence for relations among cognitive style, verbal intelligence, and subordinate category learning in early childhood raises intriguing questions concerning the role of individual characteristics throughout the continuum of 
expertise in object domains. Longitudinal research on the acquisition of expertise by individuals who vary in terms of both intelligence and cognitive style is critical for addressing these questions.

While the data from traditional categorization tasks that involve conceptual grouping and feature listing reveal much about the structure and function of concepts, they are still relatively indirect means of assessing the influence of expertise on categorization. In the next study, we asked advanced and intermediate experts direct questions pertaining to the use of features during various kinds of activities that depend on categorical knowledge, in order to evaluate differences between intermediate and advanced experts in the ways that theories impact feature use. The format of our questions and our analysis of expert responses were modeled after the knowledge assessments used by Hunn (1975) for adult experts on gulls and by $\mathrm{Ch}$ and her collaborators (Chi et al., 1989; Gobbo \& Chi, 1986) for child experts on dinosaurs.

\section{STUDY 3 \\ Verbal Reports Pertaining to Categorization}

Although verbal reports concerning mental processes have for some time been avoided because of problems associated with introspection, recent methodological and theoretical advances pertaining to verbal protocol analysis have renewed interest in this technique (Ericsson \& Crutcher, 1991; Ericsson \& Simon, 1993; Ericsson \& Smith, 1991). The analysis of experts' verbal protocols has contributed immensely to the understanding of the bases for the differences between experts and nonexperts in domains that involve problem solving or reasoning. However, there have been very few studies of expertise in object domains that have utilized this approach, perhaps because expertise in perceptually based domains lacks the overtly strategic character of skill-based domains. Nevertheless, $\mathrm{Chi}$ and her collaborators have used propositional analysis of verbal protocols to reveal important differences between child experts and novices in the way domainspecific knowledge of dinosaurs is organized (Chi et al., 1989; Chi \& Koeske, 1983; Gobbo \& Chi, 1986). It is quite possible that experts are able to hold domain-specific concepts and the relations among them in working memory while simultaneously verbalizing about their use. In this study, experts were interviewed concerning the features used when identifying, categorizing, and generating visual images of domain-specific exemplars. These data were used both to corroborate results from the triad and feature salience rating tasks of Studies 1 and 2 and to provide additional information regarding the impact of experts' theories on feature use.

\section{Method}

Subjects

The subjects included the 12 advanced experts and the 8 intermediate experts on songbirds who completed the triad task in Study 1.

\section{Materials}

Ten genera from the domain of songbirds that were native to northern Georgia (catbird, chickadee, cowbird, goldfinch, nuthatch, phoebe, swallow, tanager, vireo, wren) were discussed in reference to the four interview questions presented in Table 2

\section{Procedure}

The interview was conducted either immediately following the triad task of Study l or over the telephone within a week after completion of the triad task session. No pictures were available, and the entire session was audiotaped. Each subject within a group received a different order of the 10 bird categories. Questions were asked in the order presented in Table 2, with the exception that half of the subjects in each group received Question 3 before Question 4, and half received Question 4 before Question 3. The subjects were encouraged to take as much time as they liked in responding and to provide any additional information that they felt was relevant.

\section{Results and Discussion}

Audiotapes from the interview session were transcribed and broken down into propositions, which then were coded along dimensions specific to the expectations held for each question or pair of questions. One experimenter coded all of the verbal protocol data, and then a second experimenter (blind to the group membership of subjects) coded $25 \%$ of the data generated by the experts in each of the two groups. Reliability between the two raters was calculated by dividing the number of agreements by the number of agreements plus disagreements. Across questions 1, 3 , and 4 , reliability was .95 , and disagreements were resolved through discussion. Reliability was calculated separately for question 2 , as described below. After all of the data had been coded, the codes generated in reference to particular questions were entered into separate dBASE IV files for further analysis. Below, each of the verbal protocol topics is considered separately. Within each section, the codes developed to address the content of each response (or pair of responses) are described, and the results yielded from the coded data are presented and discussed.

\section{Question 1: Features Considered \\ Most Salient for Identification}

The first question was expected to elicit information concerning what features experts recruit when attempting to identify objects at the subordinate level. Individuals might mention behaviors (e.g., tanagers are generally high up in trees), modified parts (e.g., chickadees have a black cap and a black bib; tanagers have a uniquely shaped bill), or global features related to overall morphology, such as overall body shape and size. Although the experts were found to recruit modified parts as solutions to the triad task, it seemed possible that features pertaining to overall shape would be more relevant to identification, particularly since shape information generally is correlated with modified parts.

Individuals' responses were broken down into propositions, and each proposition was coded according to feature type. The accuracy of the experts' reports was verified through published references on songbirds; there were no 


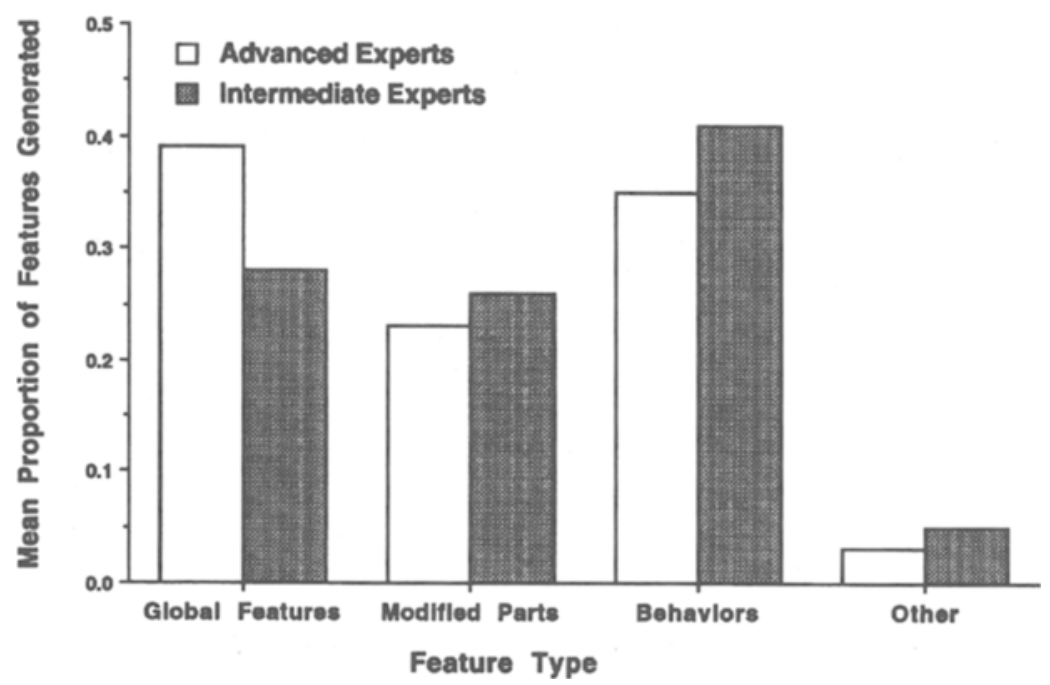

Figure 4. Mean proportions of feature types generated in response to Interview Question 1.

instances of errors in the propositions that were generated. The mean numbers of features produced across the 10 categories that were coded as global features, modified parts, and behaviors are presented in Figure 4. The frequencies of these features generated by each subject were compared across the two groups in a 2 (knowledge group) $\times 3$ (feature type) mixed ANOVA, with feature type as the within-group factor. There was no significant main effect of knowledge group, indicating that both groups of experts generated comparable numbers of propositions. The main effect of feature type was significant $[F(2,36)=4.00, p<.05]$ and was followed up through one-way repeated measures ANOVAs that were performed for each knowledge group. No main effect of feature type emerged for the advanced experts, which suggests that the three types of features were equally accessible with regard to identification. This effect was significant for the intermediate experts $[F(2,14)=$ $5.09, p<.05$ ] because significantly more behaviors were generated than were either global features $(p<.05)$ or modified parts $(p<.01)$. Behavioral features may be particularly salient for intermediate experts because of their relatively high degree of intradomain variability. Whereas overall shape differences across songbird genera are fairly subtle, behaviors pertaining to habitat, diet, and song vary considerably across different genera.

We predicted that the advanced experts would be more likely than the intermediate experts to generate global features because of advanced experts' superiority at identifying birds on the basis of shape information alone (Johnson \& Mervis, 1997). This hypothesis was confirmed through a one-tailed $t$ test $\left[M_{\mathrm{AE}}=.39, M_{\mathrm{IE}}=.28, t(18)=\right.$ $1.73, p=.05]$. The advanced experts also listed the features used as a means of differentiating between two confusable songbirds more frequently than the intermediate experts $\operatorname{did}\left[M_{\mathrm{AE}}=.31, M_{\mathrm{IE}}=.11, t(18)=2.28, p<.05\right]$. The advanced experts' recruitment of global features in identification contexts supports the idea that, as knowledge in- creases, subordinate categories begin to function as basic (Johnson \& Mervis, 1997). Sensitivity to differentiating features reflects the importance of perceptual learning to expertise acquisition in object domains. Four of the advanced experts suggested that becoming an expert on bird identification involved building skills in two distinct areas: learning to identify birds on the basis of characteristic features and learning to ignore features that are irrelevant. Additional research is necessary to determine how these two processes interact at varying points along the continuum of expertise.

\section{Question 2: Features Indicative of Canonical Images}

The second question addressed an issue raised by information provided by an airplane expert studied by Rosch et al. (1976). Rather than having a visual view of airplanes that contained features that were relevant to the typical canonical side image, this person reported having a canonical view of the undersides and engines of aircraft. This is not surprising, given that this individual acquired his expertise on airplanes through working as an aircraft mechanic. The present question was designed to address the issue of whether individuals with varying levels of expertise on songbirds, given their elaborated theories relative to characteristic behaviors, would tend to recruit behavioral features when generating visual images of exemplars. We also were interested in the relative levels of consensus on feature recruitment across the two groups. Results indicated that individuals never reported the presumably canonical side view depicted in field guides. Instead, both the advanced and the intermediate experts tended to mention location or the behavior in which birds from within that category typically were engaged. That is, experts appear to form images that contain features that pertain to those situations in which the objects are most frequently encountered. 
Table 3

Interview Question 4: Percent Agreement on Features Indicative of Canonical Images

\begin{tabular}{|c|c|c|c|}
\hline Target & Canonical Image & $\begin{array}{l}\text { Advanced } \\
\text { Experts }\end{array}$ & $\begin{array}{l}\text { Intermediate } \\
\text { Experts }\end{array}$ \\
\hline Catbird & partially concealed by thick brush & 83 & 75 \\
\hline Chickadee & actively moving about & 67 & 25 \\
\hline Cowbird & walking on the ground & 92 & 50 \\
\hline Goldfinch & feeding at a bird feeder & 58 & 38 \\
\hline Nuthatch & on the trunk of a tree & 100 & 88 \\
\hline Phoebe & perched out in the open & 92 & 75 \\
\hline Swallow & in flight & 92 & 88 \\
\hline Tanager & perched high in the tree tops & 83 & 75 \\
\hline Vireo & high in tree, feeding on insects & 33 & 38 \\
\hline Wren & in brush that is low to the ground & 58 & 38 \\
\hline
\end{tabular}

In order to assess the degree of consensus among group members on the features recruited during image formation, the descriptions provided by the experts in reference to each of the 10 target categories were combined and examined by two independent judges. Judges were instructed to read through the responses for each category and to determine the number of qualitatively different images generated among the members of the group. They then were instructed to count the number of experts within the group who generated each type of image. Reliability between the two judges was assessed by dividing the number of agreements by the total number of agreements plus disagreements. Reliability was .94 , and disagreements were resolved through discussion.

For each individual, a score assessing the degree of consensus with fellow group members was calculated for each of the 10 birds. Scores were derived by calculating the proportion of individuals within the same group who generated the same image. Proportions were averaged across the 10 categories and then compared across the two groups in a one-tailed $t$ test $\left[M_{\mathrm{AE}}=.63, M_{\mathrm{IE}}=.41, t(18)=6.36\right.$, $p<.00001]$. The advanced experts tended to converge with their peers on features indicative of images at significantly higher rates than did the intermediate experts. The most frequently generated responses for each of the 10 categories are presented in Table 3, accompanied by the percentage of the advanced and intermediate experts who produced each response. A higher consensus on the features recruited for image formation may have been a function of the advanced experts' heightened birding experience relative to that of the intermediate experts. This points to another important function of theories in feature recruitment: Theories contain information related to the frequency with which particular features (in this case, behaviors) are manifest within the domain.

\section{Questions 3 and 4: Features Selected as Bases for Similarity Relations}

The third and fourth questions concerned the similarity relations that exist among domain-specific exemplars within the knowledge base. Although morphological similarity often is a very good index of taxonomic proximity (see, e.g., Boster, 1987; Boster, Berlin, \& O'Neill, 1986;
Bulmer, 1967, 1970; Diamond, 1966; Hunn, 1975), there are some exceptions to this rule (e.g., the conflict triads from Study 1), of which only experts would be knowledgeable. Thus, both groups of experts were expected to be able to list some species exemplars for Question 3 that were not listed for Question 4, and vice versa. In addition, because of their heightened level of domain-specific knowledge, the advanced experts were expected to list greater numbers of confusable and related birds than intermediate experts did.

Confusable songbirds. The categories that the experts listed as confusable with the target category were tabulated and then coded according to the featural basis for the confusion (e.g., color, shape, behavior). The advanced experts generated significantly more confusable subordinate categories than did the intermediate experts $\left[M_{\mathrm{AE}}=.14\right.$, $\left.M_{\mathrm{IE}}=.09, t(18)=2.80, p<.01\right]$. The intermediate experts were significantly more likely than were the advanced experts to indicate that there were no birds that could be confused with the target subordinate exemplar $\left[M_{\mathrm{AE}}=.08\right.$; $\left.M_{\mathrm{IE}}=.19, t(18)=1.87, p<.05\right]$. When the features on which confusions were based were considered, color was the feature that both groups mentioned in the highest proportion of responses $(\mathrm{AE}=.35 ; \mathrm{IE}=.37)$. The intermediate experts cited behavioral features (e.g., flight, flycatching behavior) as the basis for confusion significantly more often than did advanced experts $\left[M_{\mathrm{AE}}=.08, M_{\mathrm{JE}}=.21\right.$, $t(18)=2.36, p<.05]$. These data suggest that, as expertise increases, there may be a tendency for individuals to recognize more bases for similarity among domain-specific exemplars. Although at first this seems incongruous with the results from the first question pertaining to the identification of birds, a possible explanation may involve the difference between identifying objects and thinking about conceptual relations. Identification requires that differentiating features be recruited quickly and accurately so that the most precise correct name can be generated. In contrast, the consideration of relations among concepts involves attending to higher order features that are distributed across the members of the domain.

Taxonomically related songbirds. The categories of taxonomically related songbirds that the experts listed were tabulated. The advanced experts clearly were more 
aware of taxonomic relations than the intermediate experts were. The advanced experts listed significantly more taxonomically related categories for each target than the intermediate experts did $\left[M_{\mathrm{AE}}=1.6, M_{\mathrm{IE}}=0.6, t(18)=5.19\right.$, $p<.0001]$. The intermediate experts were more likely than the advanced experts were to simply respond "I don't know" to question 4 (AE: $5 \%$ of responses, IE: $42 \%$ of responses). The advanced experts' heightened knowledge of taxonomic relations converges well with their greater tendency to recruit taxonomically related features during the triad task of Study 1.

Finally, we examined the degree of correspondence between the lists of birds generated in response to questions 3 and 4. Two sample $t$ tests revealed that, although there were no differences in the frequencies with which matching lists were generated across the two groups, the advanced experts produced significantly more nonmatching lists than did the intermediate experts $[t(18)=3.79, p<.01]$. When taxonomic relations were known, the experts were likely to indicate that there were differences between the exemplars that were perceptually confusable with the target categories and the exemplars that were closely related taxonomically to the target categories. Because the advanced experts were more knowledgeable of taxonomic relations than were the intermediate experts, this discrepancy between the two lists of categories occurred more frequently.

\section{Summary}

The results from the verbal-protocol analysis converged with and extended the findings from the previous two studies. Expert theory development involves increases in both the numbers of features known about concepts and in the degree of salience associated with those features. Theories also direct attention to particular features in particular categorization contexts. At advanced levels of expertise, subordinate-level identifications can be made on the basis of the same kinds of features that are used by novices to identify objects at the basic level. The intermediate experts tended to recruit behavioral features, whereas the advanced experts indicated that all types of features were equally relevant to identification processes. The advanced and intermediate experts were quite consistent in generating mental images that reflected the behavioral features most frequently associated with songbirds, although the degree of intragroup consensus increased as a function of knowledge. Modified part features that are indicative of relations within taxonomic genera are highly salient to experts on object domains and are most apt to be recruited in contexts involving classification or grouping. Finally, the advanced experts were aware of significantly more bases for relations among songbirds than the intermediate experts were. Since advanced experts presumably possess more elaborated theories than intermediate experts do (in part because advanced experts simply know more), advanced experts are able to recruit a wider variety of features across contexts than intermediate experts are.

\section{GENERAL DISCUSSION}

Findings from across the three studies illustrate the complex effects high levels of knowledge have on the features used in various contexts that are dependent on categorization. Expert concepts are associated with a rich array of different types of features, including those relevant to taxonomy, morphology, and behaviors. As expertise increases, features along multiple dimensions appear to increase in their degree of perceptual salience as a function of perceptual learning. Theories help to determine which of these comparably salient features are recruited within a given context. Features indicative of taxonomic relations tend to prevail in contexts involving similarity judgments or grouping. This result fits with previous findings concerning the abstract conceptual bases frequently relied upon by experts (Chi et al., 1981). Skill at detecting taxonomically relevant features may generalize even to less familiar domains, whereas other forms of expertise tend to be domain-specific. When confronted with unfamiliar exemplars from related domains, experts' theories may guide the extraction of features that align with those preferred within the familiar domain and may even amplify the degree of perceptual salience associated with those features. The advanced experts and the fish experts made decisions of similarity significantly more quickly than did the intermediate experts, which suggests that the recruitment of features in classification becomes more automated with experience. Comparisons across Studies 1 and 3 suggest that the advanced experts' more elaborated theories permitted greater flexibility in feature recruitment than did those of intermediate experts. Below, we first consider cognitive anthropological data relevant to the issue of the impact of theories on feature recruitment. We then address the mutually dependent relation between theories and perceptual information throughout the continuum of expertise.

\section{Cognitive Anthropological \\ Analyses of Feature Recruitment}

Cognitive anthropologists concerned with ethnobiology have for decades been pursuing answers to many of the same questions that cognitive psychologists have asked regarding the relation between knowledge and perception in categorization (see Malt, 1995, for a review of these literatures). The ethnobiological literature contains rich descriptions of the named categories elicited from informants (Berlin, 1992; Berlin, Breedlove, \& Raven, 1974) and the bases for relations among those named categories (Atran, 1994; Diamond, 1966). In general, a fairly high level of agreement emerges across cultures and between folk and formal scientific systems as to the features used to segment biological kinds into categories (Berlin, 1992; Hunn, 1975). These findings support the idea that features that are indicative of taxonomic relatedness are universally available and salient to humans. In support of this claim, we found that novices and experts were strikingly 
similar in their solutions to the consistent triads of Study 1 . However, it is not possible to determine whether this result obtained because the experts and the novices were actually attending to the same features or because they were attending to different features that happened to be correlated. Measuring the relative differences in response times between individuals' triad solutions based on silhouettes (in which modified parts are unavailable) and those based on realistic color pictures might help to provide an answer to this question. If experts' solution times are more greatly impeded than are novices' by the absence of modified parts, one might speculate that experts' theories were directing the recruitment of part features across multiple categorization contexts.

Although features that are indicative of categories are perceived similarly across cultures, theoretical knowledge appears to bias feature weightings across domains and across individuals possessing various levels of expertise. With regard to cross-domain differences, Boster and D'Andrade (1989) found that Aguaruna, Huambisa, and United States informants demonstrated relatively high intergroup agreement on the features recruited for differentiating birds. However, different sets of features were recruited for passerines (songbirds) and nonpasserines. Boster and D'Andrade speculate that different subdomains manifest unique correlational structures that result in different features being selected as "best" for classification within those subdomains. They suggest that humans strategically seek out features that have the greatest information value within a domain. It is likely that such a strategy would be driven by theoretical knowledge, particularly once a moderate quantity of subordinate level categories has been learned. Alternatively, individuals with varying levels of expertise on the same domain may weight features differently as a function of top-down influences on selective attention. Boster (1986) found that Aguaruna women, who typically are engaged in cultivating manioc, made finer discriminations among manioc plants than did other, less knowledgeable individuals. Similar variations in discriminability have been reported by Berlin (1992) for Aguaruna males and females with varying levels of bird knowledge.

\section{Interactions Between Intuitive Theories and Data From Perceptual Learning}

Our results give pause for speculation concerning the relative contributions of perceptual learning and theoretical knowledge throughout the continuum of expertise. Novices must begin by acquiring subordinate categories within a domain of objects and determining the bases for relations among those categories. Although related background knowledge may serve to facilitate this process (Johnson \& Mervis, 1994), it is likely that subordinate category differentiation is primarily driven by simple exposure. Perceptual learning drives selective attention to invariant features indicative of category membership; such learning may be enhanced by the presence of category names. Once a cluster of coordinate subordinates has been acquired, theories facilitate evaluation of the degree to which particular dimensions are predictive of category membership within the developing domain. Once such dimensions become weighted, perceptual differentiation may become increasingly streamlined, helping to decrease demands on processing resources and enabling subsequent theory development. This mutual interactivity between knowledge and perceptual structure fits well with Wisniewski and Medin's (1994) "tightly coupled" view of category learning.

At more advanced points along the continuum of expertise, theories may exert simultaneous effects on both the suppression and the enhancement of features. That is, theories may bias the system to weight more heavily (thereby enhancing) features that are indicative of taxonomic membership, while at the same time deemphasizing features that are less relevant to identification. In this sense, expertise promotes strategic categorization. Although categorization typically is not considered an arena for the promotion of strategies, it seems likely that experts develop heuristics for detecting invariants and higher order features and are able to generalize these processes to novel categories, even when they fall outside the domain of expertise.

Different kinds of categorization tasks promote reliance on different kinds of features. Even very young children adjust their bases for categorization decisions across tasks involving induction and tasks involving similarity relations (Deák \& Bauer, 1996). We have focused on how such adaptability in the use of features that are indicative of subordinate level categories may continue to improve at very advanced levels of knowledge. For novices, perceptual boundedness appears to be the rule. This is understandable, given that most novices know very little about the functional characteristics afforded by perceptual features that are indicative of subordinate categories. Thus, features associated with overall morphological characteristics, such as color, size, and shape, predominate in decisions of similarity. Novices' subordinate level identifications are relatively difficult to study. (Unless one happens to catch a novice on the cusp of developing expertise, subordinate category names rarely are known.) However, it seems likely that the same kinds of features recruited for similarity decisions would initially be most salient in identification (e.g., cardinals are red; dalmatians are spotted).

Intermediate and advanced experts possess denser networks of feature information and more elaborate theories concerning the relations among those features. The advanced experts had been birding for an average of 30 years and had obviously had the opportunity to encode, relate, and discuss songbird exemplars on numerous occasions. The intermediate experts generally had less experience within the domain and reported that they were engaged less often with birds on a weekly basis than were the advanced experts. Although their knowledge of songbirds was high, the intermediate experts were aware of fewer bases for relations within the songbird domain and demonstrated less flexibility in altering their recruitment of features across tasks. The advanced experts may have attained such 
high levels of automaticity in the retrieval of feature knowledge that ample processing resources were available for determining which features would be most appropriate for particular categorization tasks. Future research is needed to investigate the relative impact of information-processing resources on categorical strategy use by individuals with varying levels of domain-specific knowledge.

\section{Conclusion}

Throughout the continuum of expertise in object domains, the perception of both differentiating and higher order features remains critically important to the classification and identification of exemplars within the domain. Theories are instrumental both in the recruitment of particular features within a given context and in promoting knowledge transfer through the selection of novel features that align with those preferred within the familiar domain. The features associated with particular concepts change in strength throughout the continuum of expertise, which results in changes in identification procedures, image generation, and decisions about conceptual relations. Changes in the relative strengths of particular conceptual relations are afforded both through the learning of greater numbers of features that are indicative of particular concepts and through the noticing of features that are shared by concepts from across the domain. Quantitative variations in feature knowledge promote differing degrees of flexibility in feature recruitment across categorization tasks. Advanced experts not only know more; they know how to maximize the efficiency with which that knowledge can be used across various contexts.

\section{REFERENCES}

Alexander, J. M., Johnson, K. E., Lazar, S., \& Meyer, R. (1998) Multiple predictors of memory throughout the continuum of expertise. Manuscript submitted for publication.

Atran, S. (1994). Core domains versus scientific theories: Evidence from systematics and Itza-Maya folkbiology. In L. A. Hirschfeld \& S. A. Gelman (Eds.), Mapping the mind: Domain specificity in cognition and culture (pp. 316-340). New York: Cambridge University Press.

BERLIN, B. (1992). Ethnobiological classification: Principles of categorization of plants and animals in traditional societies. Princeton, NJ: Princeton University Press.

Berlin, B., Breedlove, D. E., \& Raven, P. H. (1974). Principles of Tzeltal plant classification: An introduction to the botanical ethnography of a Mayan-speaking people of Highland Chiapas. New York: Academic Press.

Biederman, I., \& Shiffrar, M. M. (1987). Sexing day-old chicks: A case study and expert systems analysis of a difficult perceptual-learning task. Journal of Experimental Psychology: Learning, Memory, \& Cognition, 13, 640-645.

Boster, J. [S.] (1986). Exchange of varieties and information between Aguaruna manioc cultivators. American Anthropologist, 88, 428-326.

BOSTER, J. [S.] (1987). Agreement between biological classification systems is not dependent on cultural transmission. American Anthropologist, 89, 914-920.

Boster, J. [S.], BERLIN, B., \& O'NeILL, J. (1986). The correspondence of Jívaroan to scientific ornithology. American Anthropologist, $\mathbf{8 8}$ 569-583.

Boster, J. [S.], \& D'ANDRADE, R. (1989). Natural and human sources of cross-cultural agreement in ornithological classification. American Anthropologist, 91, 132-142.

Boster, J. S., \& Johnson, J. C. (1989). Form or function: A compari- son of expert and novice judgments of similarity among fish. American Anthropologist, 91, 866-889.

BuLMER, R. N. H. (1967). Why is the cassowary not a bird? A problem of zoological taxonomy among the Karam of the New Guinea highlands. Man, 2, 1-25.

BULMER, R. N. H. (1970). Which came first, the chicken or the egghead? In J. Pouillon \& P. Maranda (Eds.), Echanges et communications: Melanges offerts à Claude Lévi-Strauss à l'occasion de son 60ème anniversaire (Vol. 2, pp. 1069-1091). The Hague: Mouton.

CAREY, S. (1985). Conceptual change in childhood. Cambridge, MA: MIT Press.

Chase, W. G., \& ERIcsson, K. A. (1981). Skilled memory. In J. R. Anderson (Ed.), Cognitive skills and their acquisition (pp. 141-189). Hillsdale, NJ: Erlbaum.

ChI, M. T. H., Feltovich, P. J., \& Glaser, R. (1981). Categorization and representation of physics problems by experts and novices. Cognitive Science, 5, 121-152.

CHI, M. T. H., GlaSer, R., \& FARR, M. J. (1988). The nature of expertise. Hillsdale, NJ: Erlbaum.

Chi, M. T. H., Hutchinson, J. E., \& Robin, A. F. (1989). How inferences about novel domain-related concepts can be constrained by structured knowledge. Merrill-Palmer Quarterly, 35, 27-62.

CHI, M. T. H., \& KoESKE, R. D. (1983). Network representation of a child's dinosaur knowledge. Developmental Psychology, 19, 29-39.

DEÁK, G. O., \& BAUER, P. J. (1996). The dynamics of preschoolers' categorization choices. Child Development, 67, 740-767.

DiAMOND, J. (1966). Classification system of primitive people. Science, 151, 1102-1104.

ERICSSON, K. A., \& CRUTCHER, R. J. (1991). Introspection and verbal reports on cognitive processes - two approaches to the study of thinking: A response to Howe. New Ideas in Psychology, 9, 57-71.

Ericsson, K. A., \& Simon, H. A. (1993). Protocol analysis: Verbal reports as data (Rev. ed.). Cambridge, MA: MIT Press.

Ericsson, K. A., \& SMith, J. (1991). Prospects and limits of the empirical study of expertise: An introduction. In K. A. Ericsson \& J. Smith (Eds.), Toward a general theory of expertise: Prospects and limits (pp. 1-38). New York: Cambridge University Press.

Gelman, S. A., \& Markman, E. M. (1986). Categories and induction in young children. Cognition, 23, 183-209.

Gelman, S. A.. \& MARKMAN, E. M. (1987). Young children's inductions from natural kinds: The role of categories and appearances. Child Development, 58, 1532-1541.

Gelman, S. A., \& Medin, D. L. (1993). What's so essential about essentialism? A different perspective on the interaction of perception, language, and conceptual knowledge. Cognitive Development, 8, 157-167.

GIBSON, E. J. (1969). Principles of perceptual learning and development. Englewood Cliffs, NJ: Prentice-Hall.

Gibson, J. J., \& GiBson, E. J. (1955). Perceptual learning: Differentiation or enrichment? Psychological Review, 62, 32-41.

Glaser, R. (1987). Thoughts on expertise. In C. Schooler \& K. W. Schaie (Eds.), Cognitive functioning and social structure over the lifecourse (pp. 81-94). Norwood, NJ: Ablex.

GoBBo, C., \& CHI, M. (1986). How knowledge is structured and used by expert and novice children. Cognitive Development, 1, 221-237.

Hirschfeld, L. A., \& Gelman, S. A. (1994). Toward a topography of mind: An introduction to domain specificity. In L. A. Hirschfeld \& S. A. Gelman (Eds.), Mapping the mind: Domain specificity in cognition and culture (pp. 3-35). New York: Cambridge University Press.

HunN, E. (1975). Cognitive processes in folk ornithology: The identification of gulls (Working Paper No. 42). Berkeley: University of California, Language Behavior Research Laboratory.

JoHnson, K. E., \& EILERs, A. T. (1996, March). Effects of knowledge and development on the extension and evolution of subordinate categories. Paper presented at the Conference on Human Development, Birmingham, AL.

Johnson, K. E., \& Mervis, C. B. (1994). Microgenetic analysis of first steps in children's acquisition of expertise on shorebirds. Developmental Psychology, 30, 418-435.

Johnson, K. E., \& Mervis, C. B. (1997). Effects of varying levels of expertise on the basic level of categorization. Journal of Experimental Psychology: General, 126, 248-277. 
JONES, S. S., \& SMITH, L. B. (1993). The place of perception in children's concepts. Cognitive Development, 8, 113-139.

KarmilofF-SMith, A. (1992). Beyond modularity: A developmental perspective on cognitive science. Cambridge, MA: MIT Press.

KEIL, F. C. (1989). Concepts, kinds, and cognitive development. Cambridge, MA: MIT Press.

MALT, B. C. (1995). Category coherence in cross-cultural perspective. Cognitive Psychology, 29, 85-148.

MEdin, D. L. (1989). Concepts and conceptual structure. American Psychologist, 44, 1469-1481.

Medin, D. L., Goldstone, R. L., \& Gentner, D. (1993). Respects for similarity. Psychological Review, 100, 254-278.

Medin, D. L., Lynch, E. B., Coley, J. D., \& Atran, S. (1997). Categorization and reasoning among tree experts: Do all roads lead to Rome? Cognitive Psychology, 32, 49-96.

Mervis, C. B., Johnson, K. E., \& Mervis, C. A. (1994). Acquisition of subordinate categories by three-year-olds: The roles of attribute salience, linguistic input, and child characteristics. Cognitive Development, 9, 211-234.

MurPHy, G. L., \& MEDIN, D. L. (1985). The role of theories in conceptual coherence. Psychological Review, 92, 289-316.

MURPHY, G. L., \& WRIGHT, J. C. (1984). Changes in conceptual structure with expertise: Differences between real-world experts and novices. Journal of Experimental Psychology: Learning, Memory, \& Cognition, 10, 144-155.

NeISSER, U. (1987). From direct perception to conceptual structure. In U. Neisser (Ed.), Concepts and conceptual development: Ecological and intellectual factors in categorization (pp. 11-24). New York: Cambridge University Press.

Norman, G. R., Brooks, L. R., Coblentz, C. L., \& BabCook, C. J. (1992). The correlation of feature identification and category judgments in diagnostic radiology. Memory \& Cognition, 20, 344-355.

PICK, A. D. (1965). Improvement of visual and tactual form discrimination. Journal of Experimental Psychology, 69, 331-339.

Proctor, R. W., \& DutTA, A. (1995). Skill acquisition and human performance. Thousand Oaks, CA: Sage.

Rosch, E., Mervis, C. B., Gray, W. D., Johnson, D. M., \& BoyesBraem, P. (1976). Basic objects in natural categories. Cognitive Psychology, 8, 382-439.

ScotT, P., \& Mervis, C. B. (1993, March). The continuum of child expertise: The dinosaur domain. Paper presented at the Biennial Meeting of the Society for Research in Child Development, New Orleans.
Smith, J. D., \& Kemler-Nelson, D. G. (1984). Overall similarity in adults' classification: The child in all of us. Journal of Experimental Psychology: General, 113, 137-159.

STROss, B. (1973). Acquisition of botanical terminology by Tzeltal children. In M. S. Edmonson (Ed.), Meaning in Mayan languages (pp. 107141). The Hague: Mouton.

TANAKA, J. W., \& TAYLOR, M. (1991). Object categories and expertise: Is the basic level in the eye of the beholder? Cognitive Psychology, 23, 457-482.

Voss, J. F., \& Post, T. A. (1988). On the solving of ill-structured problems. In M. T. H. Chi, R. Glaser, \& M. J. Farr (Eds.), The nature of expertise (pp. 261-285). Hillsdale, NJ: Erlbaum.

WISNIEWSKI, E. J., \& MEDIN, D. L. (1994). On the interaction of theory and data in concept learning. Cognitive Science, 18, 221-281.

\section{NOTES}

1. Although it is possible that differences between expert and novice performance in the less familiar domain arose because of differences in age or level of motivation, we consider this unlikely. Parallel transfer effects have been found more recently in a study of subordinate category extension patterns involving adult dinosaur experts (Johnson \& Eilers, 1996). In this study, dinosaur experts performed better than novices on trials involving unfamiliar shorebird exemplars, even though the groups did not differ in terms of age or the amount that they were paid for their participation.

2. Identical patterns of results were found for response times to consistent triads. The intermediate bird experts solved these triads more slowly than did any other group $(p<.05)$.

3 . The two advanced experts from Study 1 who provided this information solved conflict triads involving these birds by pairing the bunting and the grosbeak. These solutions were coded as taxonomically based. No other experts generated this solution.

4. Although one might question whether the experts actually were rating perceptibility or some other aspect of feature importance (such as the degree to which feature $X$ varied within the domain), we are convinced that the obviousness of features was rated. The experts and novices also were asked to rate high and low salience features of bicycles, dogs, scissors, and shoes for which adult salience ratings had been previously collected (Mervis et al., 1994). No group differences emerged in the ratings of these features, which suggests that the experts and novices were rating features in the same way. 
APPENDIX A

Features Indicative of Morphological as Opposed to Taxonomic Relations Among Songbirds

\begin{tabular}{|c|c|c|}
\hline Triad Composition & $\begin{array}{c}\text { Morphological } \\
\text { Features }\end{array}$ & $\begin{array}{c}\text { Taxonomic } \\
\text { Features }\end{array}$ \\
\hline $\begin{array}{l}\text { 1. acadian flycatcher } \\
\text { vermillion flycatcher } \\
\text { scarlet tanager }\end{array}$ & coloration & $\begin{array}{l}\text { bill shape } \\
\text { posture }\end{array}$ \\
\hline $\begin{array}{l}\text { 2. barn swallow } \\
\text { rough-winged swallow } \\
\text { orchard oriole }\end{array}$ & coloration & $\begin{array}{l}\text { bill shape } \\
\text { rounded head shape }\end{array}$ \\
\hline $\begin{array}{l}\text { 3. black-and-white warbler } \\
\text { hooded warbler } \\
\text { American goldfinch }\end{array}$ & coloration & bill shape \\
\hline $\begin{array}{l}\text { 4. black-throated blue warbler } \\
\text { blue-winged warbler } \\
\text { yellow-throated vireo }\end{array}$ & coloration & $\begin{array}{l}\text { bill shape } \\
\text { posture }\end{array}$ \\
\hline $\begin{array}{l}\text { 5. blue grosbeak } \\
\text { pine grosbeak } \\
\text { purple finch }\end{array}$ & coloration & bill shape \\
\hline $\begin{array}{l}\text { 6. great-crested flycatcher } \\
\text { vermillion flycatcher } \\
\text { scarlet tanager }\end{array}$ & coloration & $\begin{array}{l}\text { bill shape } \\
\text { posture }\end{array}$ \\
\hline $\begin{array}{l}\text { 7. red-eyed vireo } \\
\text { yellow-throated vireo } \\
\text { blue-winged warbler }\end{array}$ & coloration & $\begin{array}{l}\text { bill shape } \\
\text { posture }\end{array}$ \\
\hline $\begin{array}{l}\text { 8. varied thrush } \\
\text { hermit thrush } \\
\text { fox sparrow }\end{array}$ & coloration & $\begin{array}{l}\text { bill shape } \\
\text { eye shape }\end{array}$ \\
\hline
\end{tabular}




\section{APPENDIX B}

Features Indicative of Morphological as Opposed to Taxonomic Relations Among Tropical Fish

\begin{tabular}{|c|c|c|c|}
\hline & Triad Composition & $\begin{array}{l}\text { Morphological } \\
\text { Features }\end{array}$ & $\begin{array}{c}\text { Taxonomic } \\
\text { Features }\end{array}$ \\
\hline & $\begin{array}{l}\text { Argentine pearl fish } \\
\text { White's pearl fish } \\
\text { pearl danio }\end{array}$ & $\begin{array}{l}\text { body shape } \\
\text { coloration }\end{array}$ & $\begin{array}{l}\text { tail shape } \\
\text { ventral fin shape }\end{array}$ \\
\hline 2. & $\begin{array}{l}\text { cardinal tetra } \\
\text { black neon tetra } \\
\text { three-lined pencilfish }\end{array}$ & coloration & $\begin{array}{l}\text { fin orientation } \\
\text { dorsal fin shape }\end{array}$ \\
\hline & $\begin{array}{l}\text { cherry barb } \\
\text { striped barb } \\
\text { zebra danio }\end{array}$ & striped pattern & $\begin{array}{l}\text { dorsal fin shape } \\
\text { tail shape }\end{array}$ \\
\hline & $\begin{array}{l}\text { cherry barb } \\
\text { tiger barb } \\
\text { common glassfish }\end{array}$ & body shape & dorsal fin shape \\
\hline & $\begin{array}{l}\text { chocolate gourami } \\
\text { pearl gourami } \\
\text { jewel cichlid }\end{array}$ & $\begin{array}{l}\text { size } \\
\text { spotted pattern }\end{array}$ & $\begin{array}{l}\text { fin shape } \\
\text { mouth shape }\end{array}$ \\
\hline & $\begin{array}{l}\text { kuhli loach } \\
\text { clown loach } \\
\text { bumblebee goby }\end{array}$ & $\begin{array}{l}\text { body shape } \\
\text { striped pattern }\end{array}$ & mouth shape \\
\hline & $\begin{array}{l}\text { pearl danio } \\
\text { zebra danio } \\
\text { striped barb }\end{array}$ & striped pattern & mouth shape \\
\hline 8. & $\begin{array}{l}\text { red swordtail } \\
\text { green swordtail } \\
\text { black-lined rainbow fish }\end{array}$ & coloration & fin shape \\
\hline 9. & $\begin{array}{l}\text { rummy-nose tetra } \\
\text { featherfin tetra } \\
\text { tinfoil barb }\end{array}$ & $\begin{array}{l}\text { body shape } \\
\text { coloration }\end{array}$ & $\begin{array}{l}\text { fin shape } \\
\text { size }\end{array}$ \\
\hline 10. & $\begin{array}{l}\text { tiger barb } \\
\text { rosy barb } \\
\text { redbelly notho }\end{array}$ & $\begin{array}{l}\text { body shape } \\
\text { coloration }\end{array}$ & $\begin{array}{l}\text { dorsal fin shape } \\
\text { tail fin shape } \\
\text { ventral fin shape }\end{array}$ \\
\hline
\end{tabular}

(Manuscript received September 25, 1996; revision accepted for publication December 27, 1996.) 